\title{
Numerical Simulation of a Periodic Quasi-Switching Mode of Flow around a Conical Dimple with a Slope Angle of 10 Degrees on the Wall of a Narrow Channel Using URANS
}

\author{
Sergey Isaev $\left.{ }^{1,2} \mathbb{(}\right)$, Dmitry Nikushchenko ${ }^{1} \mathbb{D}$, Alexandr Sudakov ${ }^{2}$, Nikita Tryaskin ${ }^{1, * \mathbb{C}}$, Ann Egorova ${ }^{1}$, \\ Leonid Iunakov $^{3}$, Alexandr Usachov ${ }^{4}$ and Valery Kharchenko ${ }^{2}$ \\ 1 Research Department, Saint-Petersburg State Marine Technical University, Lotsmanskaya Str., 3, \\ 190121 Saint-Petersburg, Russia; isaev3612@yandex.ru (S.I.); ndmitry@list.ru (D.N.); \\ egorova.anna@mail.ru (A.E.) \\ 2 Research Department, Saint-Petersburg State University of Civil Aviation, Pilots Str. 38, \\ 196210 Saint-Petersburg, Russia; sudakov-1950@mail.ru (A.S.); val-spb@mail.ru (V.K.) \\ 3 Research Department, Baltic State Technical University, 1-a Krasnoarmejskaya Str., 1, \\ 190005 Saint-Petersburg, Russia; yunakovlp@mail.ru \\ 4 Research Department, N.E. Zhukovskii Central Aerohydrodynamic Institute, Radio Str., 17, \\ 107005 Moscow, Russia; usachov_al@mail.ru \\ * Correspondence: nikita.tryaskin@smtu.ru
}

\section{check for} updates

Citation: Isaev, S.; Nikushchenko, D.; Sudakov, A.; Tryaskin, N.; Egorova,

A.; Iunakov, L.; Usachov, A.;

Kharchenko, V. Numerical Simulation of a Periodic Quasi-Switching Mode of Flow around a Conical Dimple with a Slope Angle of 10 Degrees on the Wall of a Narrow Channel Using URANS. Fluids 2021, 6, 385. https:// doi.org/10.3390/fluids6110385

Academic Editor: Markus Klein

Received: 21 August 2021

Accepted: 18 October 2021

Published: 26 October 202

Publisher's Note: MDPI stays neutral with regard to jurisdictional claims in published maps and institutional affiliations.

Copyright: (C) 2021 by the authors Licensee MDPI, Basel, Switzerland. This article is an open access article distributed under the terms and conditions of the Creative Commons Attribution (CC BY) license (https:// creativecommons.org/licenses/by/ $4.0 /)$
Abstract: The applicability of the solution of the unsteady Reynolds-averaged Navier-Stokes equations (URANS) for the numerical simulation of the periodic quasi-switching regime of vortex generation and heat transfer in a deep conical dimple with a slope angle of $10^{\circ}$ on the wall of a narrow channel is substantiated. To calculate the turbulent regime, the model of shear stress transfer by Menter 2003, modified taking into account the influence of the curvature of streamlines within the framework of the Rodi-Leshziner-Isaev approach, is used. At Reynolds number $R e=10^{4}$, the oscillation period of the transverse $R_{z}$ and longitudinal forces $R_{x}$, as well as the total heat transfer $N u_{m m}$ to the control section of the heated channel wall with a dimple, is set equal to 60 , which corresponds to the Strouhal number $S t=0.0167$. Computer visualization of swirling jet-vortex flows demonstrates focus-type sources on the side faces of the dimple. In the self-oscillating mode, a two-cell vortex system is formed with different intensities at the oscillation period $R_{z}$. Periodic changes in friction, Nusselt numbers and temperature are recorded in the longitudinal and transverse median sections of the dimple and reflect the oscillations of the vortex structure from left to right and from right to left. The formation of a fan jet is shown, which oscillates relative to the plane of longitudinal symmetry, causing a redistribution of power and thermal loads.

Keywords: jet-vortex generation; heat transfer; conical dimple; channel; simulation; URANS; quasi-switching mode

\section{Introduction}

The problem of modeling self-oscillating modes of unsteady separated flow around objects with periodic vortex formation is one of the actual problems of hydromechanics. Self-oscillations of vortex structures are accompanied by periodic changes in local characteristics, as well as local and integral force loads on objects. Positive experience has been accumulated in the numerical prediction of the characteristics of turbulent flow based on the solution of unsteady Reynolds-averaged Navier-Stokes equations (URANS) [1-6], modeling by Large Eddy Simulation (LES) [7-10] and Direct Numerical Simulation (DNS) [11] The calculation results are in good agreement with the data of physical experiments [12,13]. It is important to note that using URANS it is possible to identify the process of the formation of unsteady spatial jet-vortex structures and determine the effect of the height of the protrusion on it. 
The alternating descent of vortices from the side faces of a cube is similar to the formation of a vortex street behind a circular cylinder in the case of a periodic two-dimensional flow (see [14]). As the height of the protrusion increases, the vertical swinging of the vortex structures intensifies.

Spatial internal flows with periodic vortex formation in channels with a transverse circular cavity are characterized by a lower intensity of return and secondary flows in comparison with external cyclic flow around the ridges [4]. A circular cavity on the wall of a narrow channel induces longitudinal transverse oscillations of vortex structures in it. The lateral load on the side wall $R_{z}$ conform a close to sinusoidal law from time to time.

As shown by experimental studies [15], when flowing around a hemispherical dimple on a plate, periodic jet-vortex structures are formed in it, alternately forming on the side slopes of the dimple. The same switching modes are recorded in narrow channels with a spherical dimple with a sharp edge [16]. The use of the LES methodology made it possible to numerically reproduce the periodic regimes of vortex formation in a deep spherical dimple on the channel wall [17].

This study is a continuation of the numerical study of the flow around a conical dimple on the wall of a narrow channel when the slope angle changes from $10^{\circ}$ to $60^{\circ}$ [18] with an emphasis on modeling the quasi-switching regime.

\section{Problem Statement and Solution Method}

The configuration of a narrow plane-parallel channel with a conical dimple on the bottom wall, as shown in Figure 1, that has been tested in a number of studies is considered. It is a computer analogue of the channel of experimental stands [16-18]. As characteristic parameters, the dimple spot diameter $D$ the mean bulk velocity $U$, and the $D / U$ ratio (unit of time) are chosen. The slope angle of the dimple is set equal to $10^{\circ}$, i.e., and the dimple is close to cylindrical in shape. The relative depth of the dimple is quite large and amounts to 0.233 . The height of the narrow channel is taken to be 0.33 , and the channel width is 2.5 . The turbulence intensity is set close to the experimental one $(1-5 \%)$, and the scale of turbulence is taken on the order of the characteristic size. The relative radius of rounding of the edge of the dimple is 0.025 . The Reynolds number in the stabilized section of the air flow in the channel is taken equal to $10^{4}$. The Prandtl number is 0.71 .

The computational domain covers the space bounded by the walls of a narrow channel with a length of 6.7, as shown in Figure 1. The inlet flow boundary with the profiles of the characteristics of a fully developed turbulent flow is located at a distance of 1.4 from the projection of the dimple center onto the plane of the bottom wall, which is taken as the center of the Cartesian $x, y, z$ coordinate system. In the outlet section of the channel, "soft" boundary conditions are set-conditions for the continuation of the solution to the boundary [19] — and no-slip conditions are set on the washed channel walls.

To solve the problem, a multi-block computational grid is used, containing about one million cells [20]. For its composition, the grid consists of four different-scale fragments, Figure 1b:

1. a rectangular channel (with a minimum step along the longitudinal coordinate- -0.08 and along the transverse coordinate-0.07) mesh with thickening towards the wall, designed to display the flow within the channel;

2. detailed region, designed for detailing the flow in the near wake of the dimple, a Cartesian grid covering the vicinity of the dimple with dimensions of $3.3 \times 0.1 \times 1.7$ (with the front boundary at a distance of 0.44 from the center);

3. a cylindrical, curvilinear, elliptical mesh, adjacent to the irradiated wall, matched to the surface of a spherical hole, thickening towards the wall;

4. an oblique mesh covering the axis of a cylindrical hole mesh-patches $0.2 \times 0.2$ in size (see [18]). 


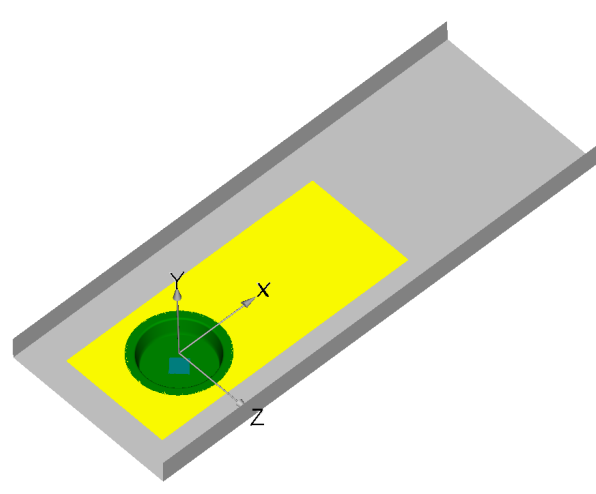

(a)

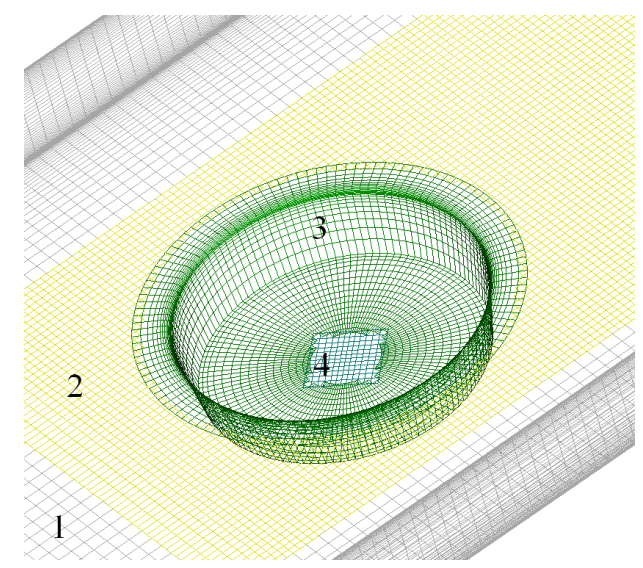

(b)

Figure 1. Section of a plane-parallel channel with a conical dimple on the heated wall (a) with the Cartesian $x, y, z$ coordinate system and multi-block grids in the vicinity of the dimple (b). 1 -channel grid (CG); 2-refined Cartesian grid (wake grid-WG) covering the dimple; 3-O-shaped grid Ring inside the dimple; $4-$ Rec grid-axial patch. The upper wall of the channel is removed. Reproduced from [20], published by MDPI 2021.

The wall step is chosen equal to $10^{-4}$. The channel mesh contains $83 \times 69 \times 87$ cells, and the detailed rectangular mesh surrounding the spherical hole contains $132 \times 31 \times 59$ cells. For detailed region 2, the minimum values of the longitudinal and transverse spacing are 0.03 . The cylindrical elliptical mesh, matched to the hole, contains $108 \times 53 \times 103$ cells, and the oblique mesh covering the axis contains $21 \times 53 \times 21$ cells.

The acceptability of URANS for calculating spatial separated flows is substantiated in a number of numerical studies, including calculations of the flow around a cube in a channel [13].

The thermal problem is solved separately from the hydrodynamic one (in this case, the effects of natural convection are not taken into account). A fully developed air flow in the inlet section of the channel is assumed to be isothermal with a characteristic temperature $T_{\text {ref }}=293 \mathrm{~K}$. The lower washed-out dimpled channel wall is heated and maintained at a constant heat flux $q$. The heat flux $q$ is recalculated into a dimensionless form by the formula

$$
\bar{q}=\frac{q}{\lambda \operatorname{PrRe} T_{r e f} / D}
$$

and is taken to be of the order of $3.408 \cdot 10^{-5}$.

Here $\lambda$ is the thermal conductivity of air. The side walls of the channel are adiabatic, and the upper one is isothermal with the temperature $T_{\text {ref }}$ taken as the dimensionless scale. At the outlet of the channel, soft boundary conditions are set for $T$.

Numerical solution of unsteady Reynolds-averaged Navier-Stokes equations (URANS) and energy is based on the concept of splitting into physical processes [19] and uses the SIMPLEC pressure correction procedure and multi-block structured grids with their partial overlap [4]. The equations of motion are closed using a semiempirical modified shear stress transport (MSST) model, which was proposed in 2003 by Menter [21,22], taking into account the influence of the curvature of streamlines. The approach of Rodi-Leschziner-Isaev [23] to the 2003 MSST correction consists of introducing a correction function $f_{c}=1 /\left(1+C_{c} \times R i_{t}\right)$ into the eddy viscosity coefficient $\mu_{t}$, where an additional constant $C_{c}=0.02$, and the product $f_{c} \times C_{\mu}$ is superimposed limitation $0.02<f_{c} \times C_{\mu}<0.15$ (in the standard $k-\epsilon$ model $[24,25]$, the semiempirical constant in the expression for the vortex viscosity is $C_{\mu}=0.09$ ). It is of fundamental importance to determine the turbulent Richardson number for spatial flows (for two-dimensional flows, it depends on the local flow velocity and the 
radius of curvature of the streamline, as shown in [25]) and modify it for three-dimensional flows, as shown in [26,27].

The developed methodology is parallelized in relation to cluster (multicore and multiprocessor) systems and generalized to the case of unstructured grids. Like [4], the generalized transport equation is written in increments of the dependent variables. The explicit part of the equation is discretized according to second-order approximation schemes (upwind scheme with Leonard's quadratic interpolation [28] for convective terms of the equations of momentum; TVD scheme [29] for convective terms of equations of turbulence characteristics; a central difference scheme for diffusion terms). The implicit part is represented using a one-way difference upstream scheme. The use of centered grids with the placement of dependent variables in the centers of the computational cells leads to the need to monotonize the pressure field due to the introduction of the Rhie-Chow correction [30,31]. The method for solving algebraic equations is the preconditioned BiCGSTAB [32] with an algebraic multigrid accelerator from the Demidov library (amgcl) [33] for the pressure correction and ILU0 for the remaining variables. The developed VP2/3 (Velocity-Pressure, 2D/3D) package uses multiblock computing technologies (MBT) described in [4]. They are based on a set of multi-scale, tiered and intersecting structured grids, consistent with the structural elements of the physical problem of the corresponding scales. In two rows of near-boundary cells of each of the overlaping or overset grids, the parameters are determined using linear interpolation [4].

Thus, when integrating the equations, a method of the second order of approximation in spatial variables and of the first order in time with dimensionless time step of 0.02 is used. For the initial state of the air flow in the channel with a conical dimple, the velocity field on the stabilized section of the plane-parallel channel is taken. In this case, at $t<0$, it is assumed that there is no air movement inside the dimple. The computational process ends when the periodic mode of changing the local and integral parameters, including the shear force $R_{z}$, acting on the control section with a dimple $2.5 \times 1.5$ in size with a center shift downstream by 0.5 , is reached. In this paper, local and integral characteristics are analyzed for the period of oscillations $R_{z}$.

\section{Analysis of the Obtained Results}

Figures 2-7 shows some of the results.

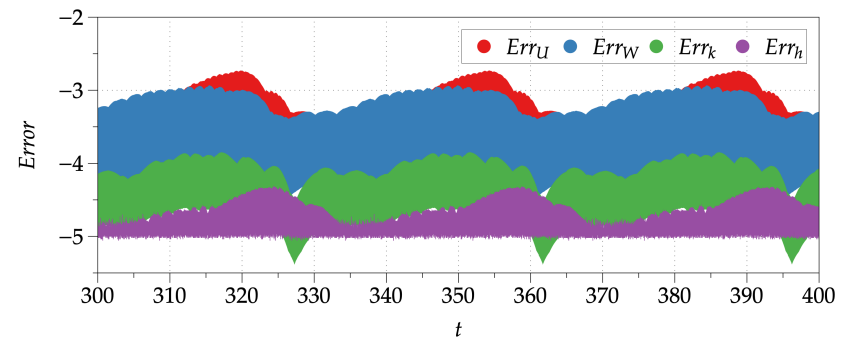

(a)

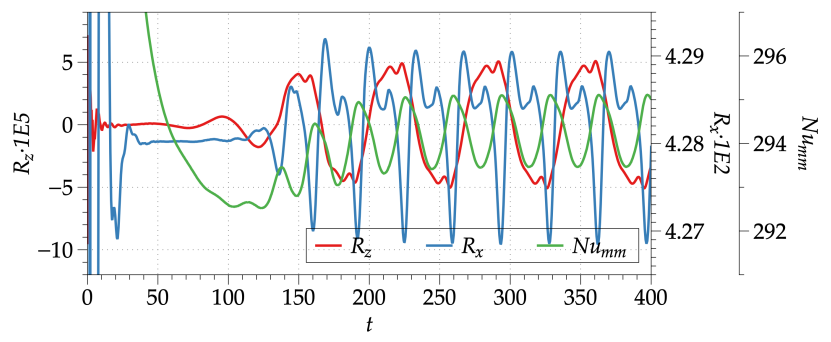

(b)

Figure 2. Time dependences of the maximum increments of the longitudinal $E r r_{U}$ and transverse $E r r_{W}$ components of the velocity, turbulence energy Err ${ }_{k}$, enthalpy (a) acting on the control section with a dimple of transverse $R_{z}(5)$ and longitudinal $R_{x}$ forces, as well as the total heat transfer $N u_{m m}(\mathbf{b})$. 


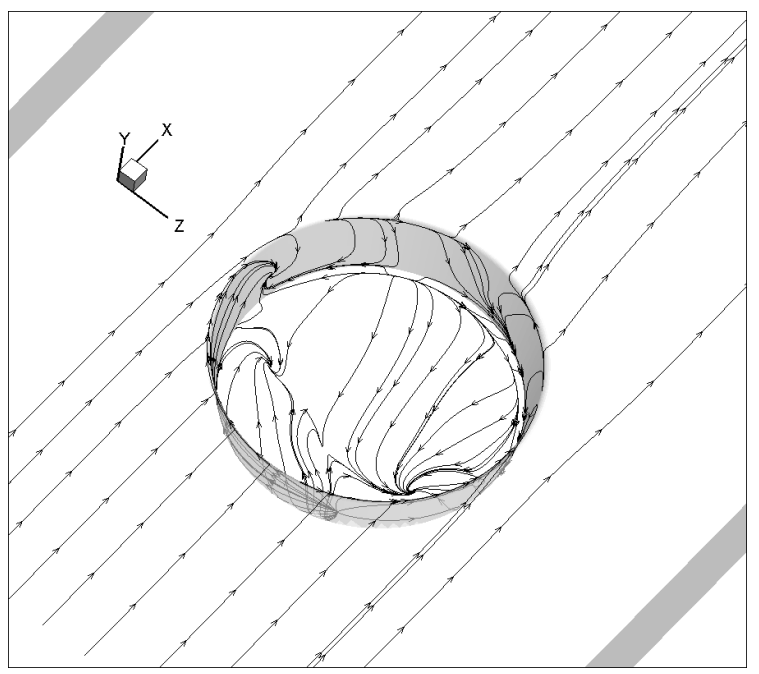

(a)

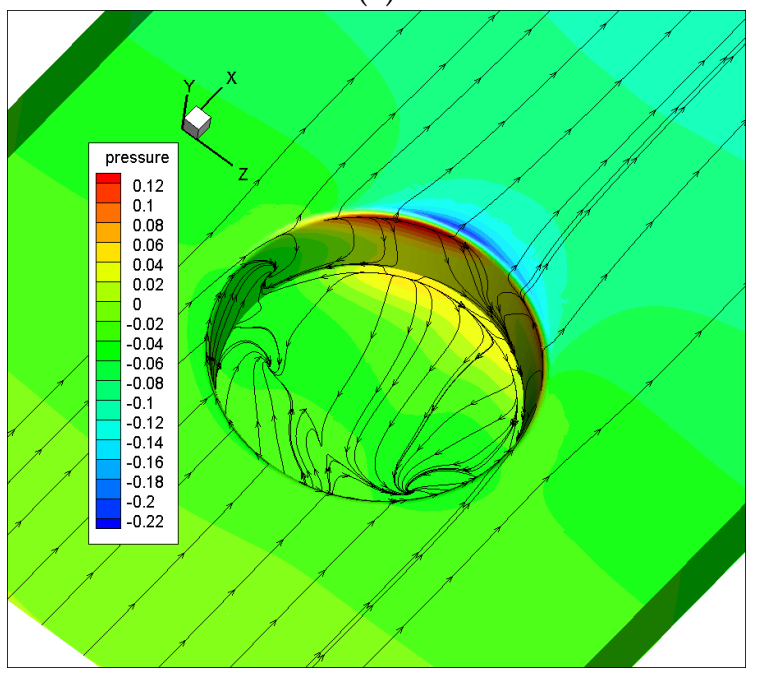

(c)

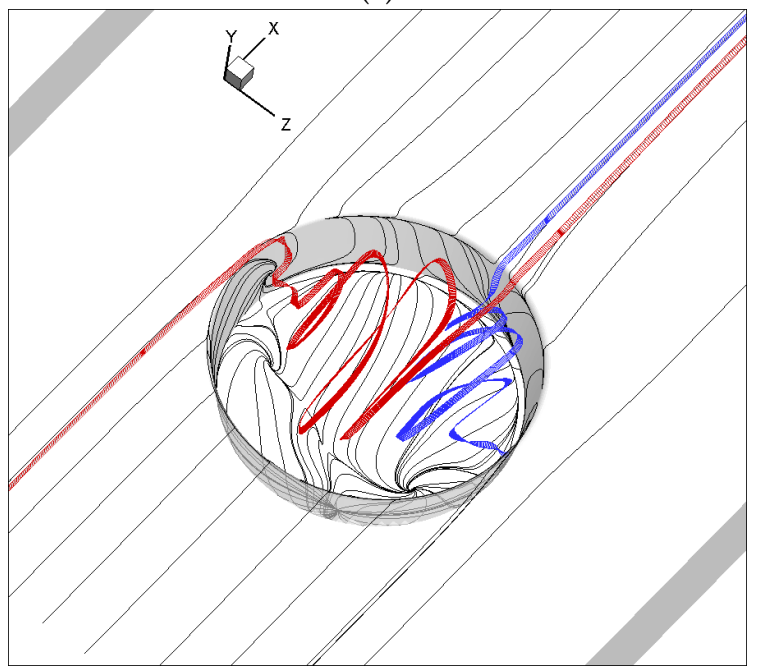

(e)

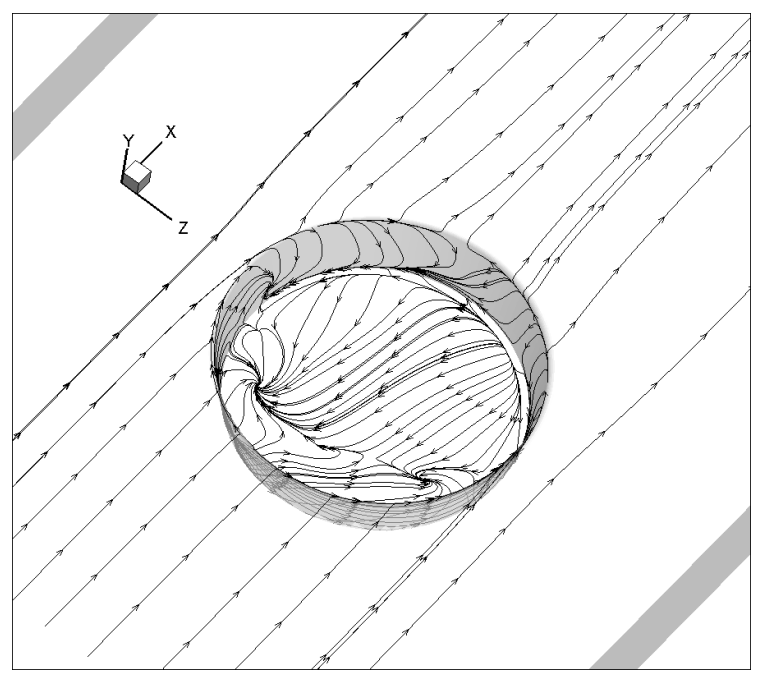

(b)

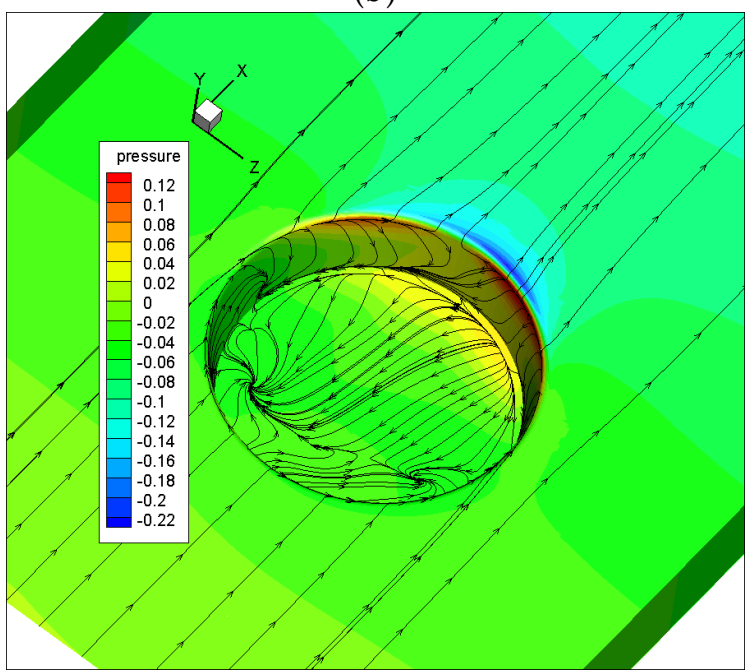

(d)

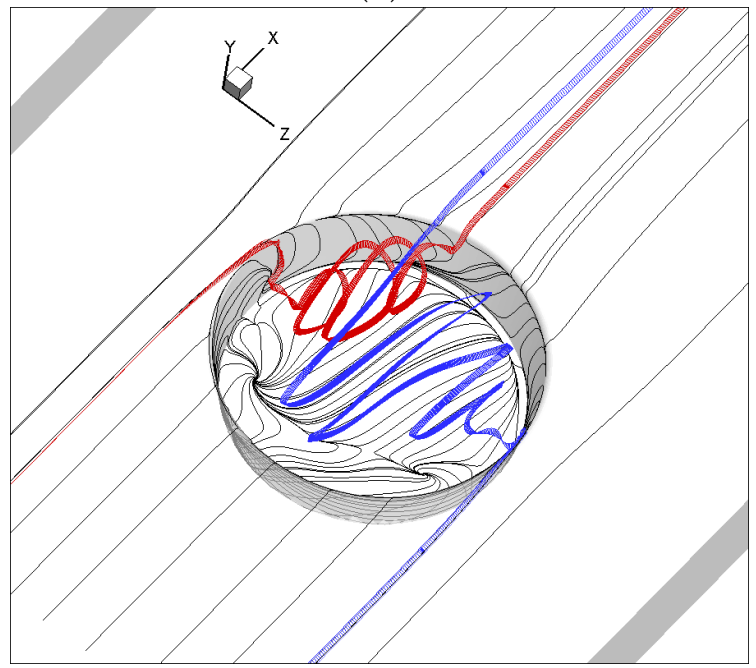

(f)

Figure 3. Patterns of spreading $(\mathbf{a}, \mathbf{b})$, pressure fields (c,d) and jet-vortex structures $(\mathbf{e}, \mathbf{f})$ at times $t=258(\mathbf{a}, \mathbf{c}, \mathbf{e})$ and $291(\mathbf{b}, \mathbf{d}, \mathbf{f})$, corresponding to $R_{z}=R_{z_{\min }}$ and $R_{z_{\max }}$, respectively. 


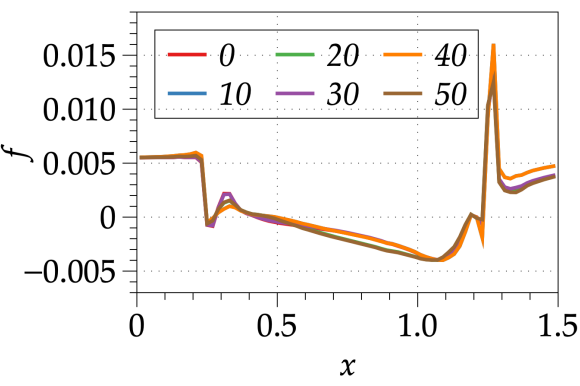

(a)

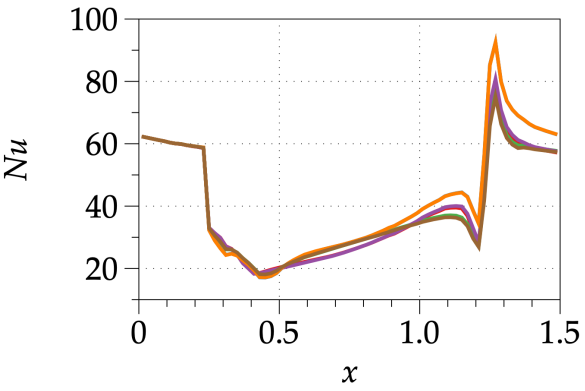

(c)

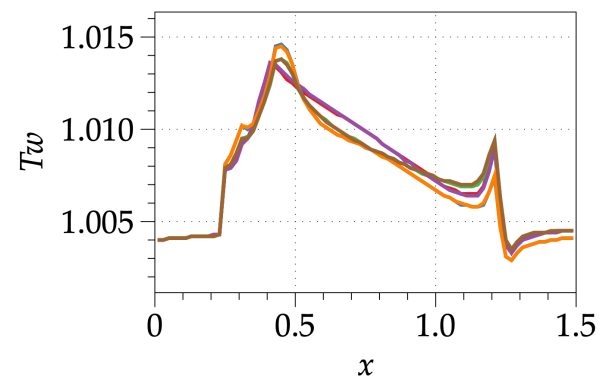

(e)

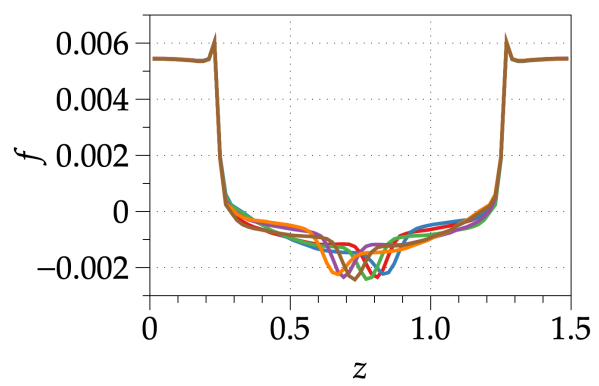

(b)

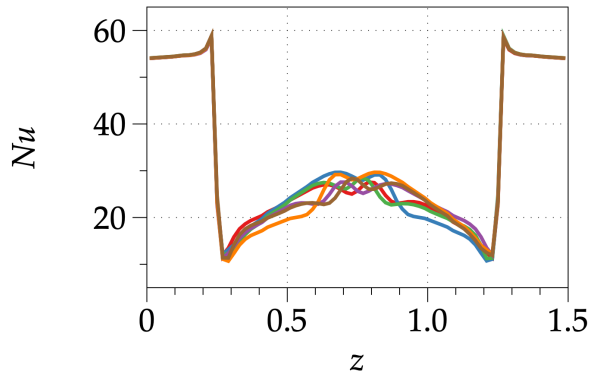

(d)

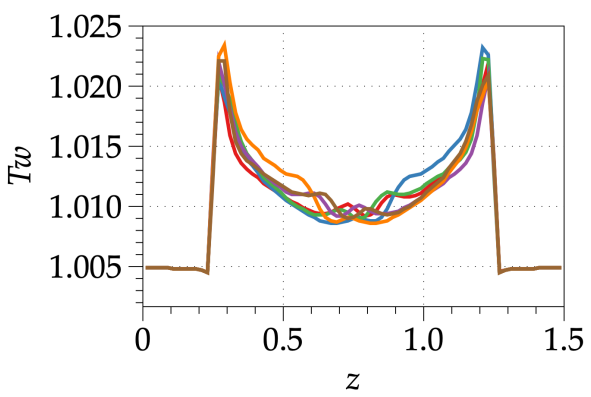

(f)

Figure 4. Time evolution $t^{*}$ on the oscillation period $T$ of the transverse force $R_{z}$ of the distributions of dimensioned friction $f(\mathbf{a}, \mathbf{b})$, Nusselt number $N u(\mathbf{c}, \mathbf{d})$ and dimensioned wall temperature $T_{w}(\mathbf{e}, \mathbf{f})$ in the median longitudinal $(\mathbf{a}, \mathbf{c}, \mathbf{e})$ and transverse $(\mathbf{b}, \mathbf{d}, \mathbf{f})$ cross-sections.

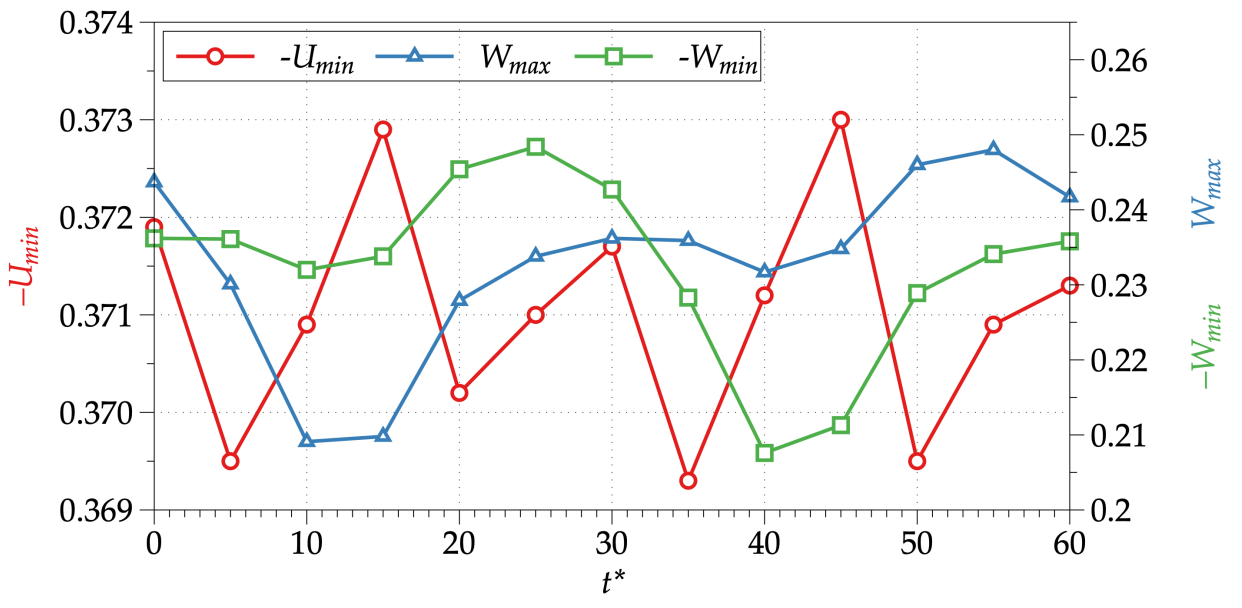

Figure 5. Dependence on $t^{*}$ of extreme values $-U_{\min }, W_{\max },-W_{\min }$. 


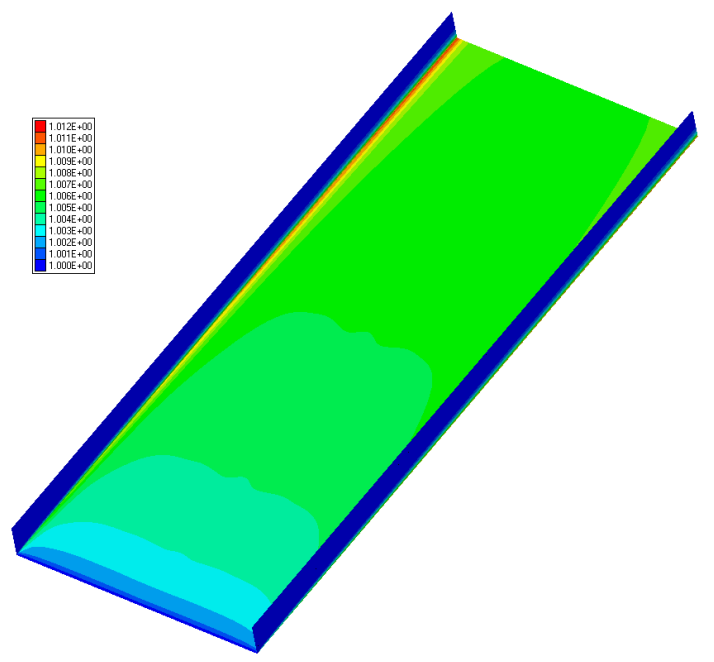

(a)

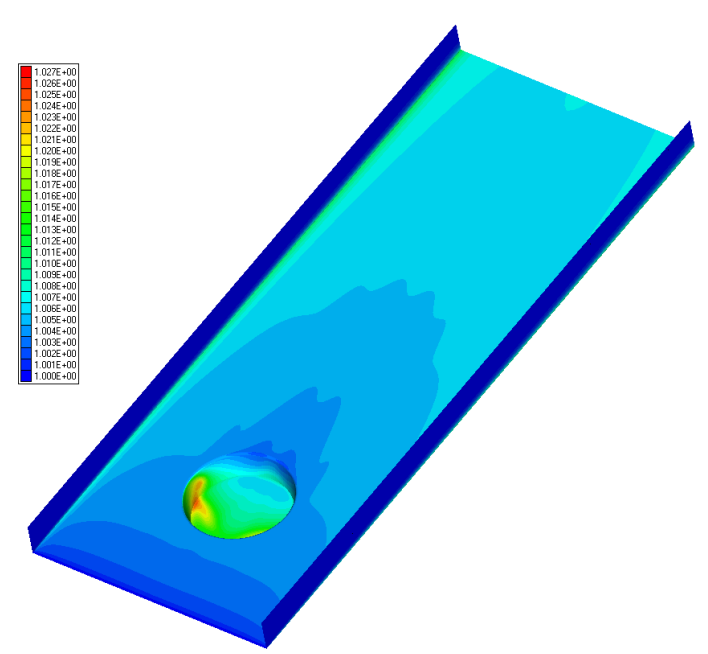

(b)

Figure 6. Comparison of temperature distributions of heated walls of plane-parallel (a) and dimpled (b) channels.
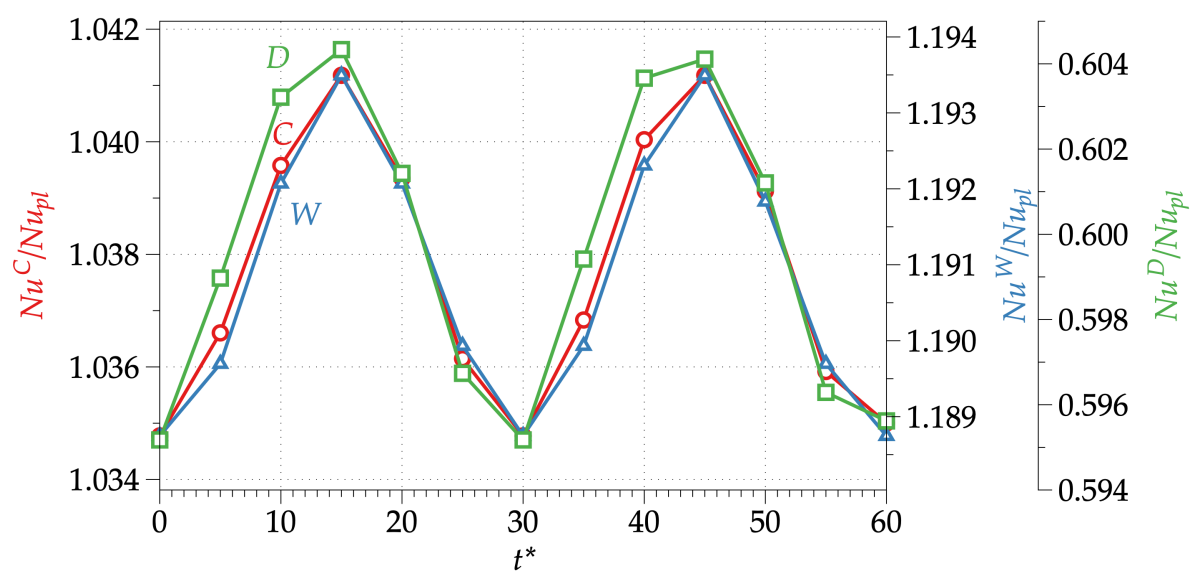

(a)

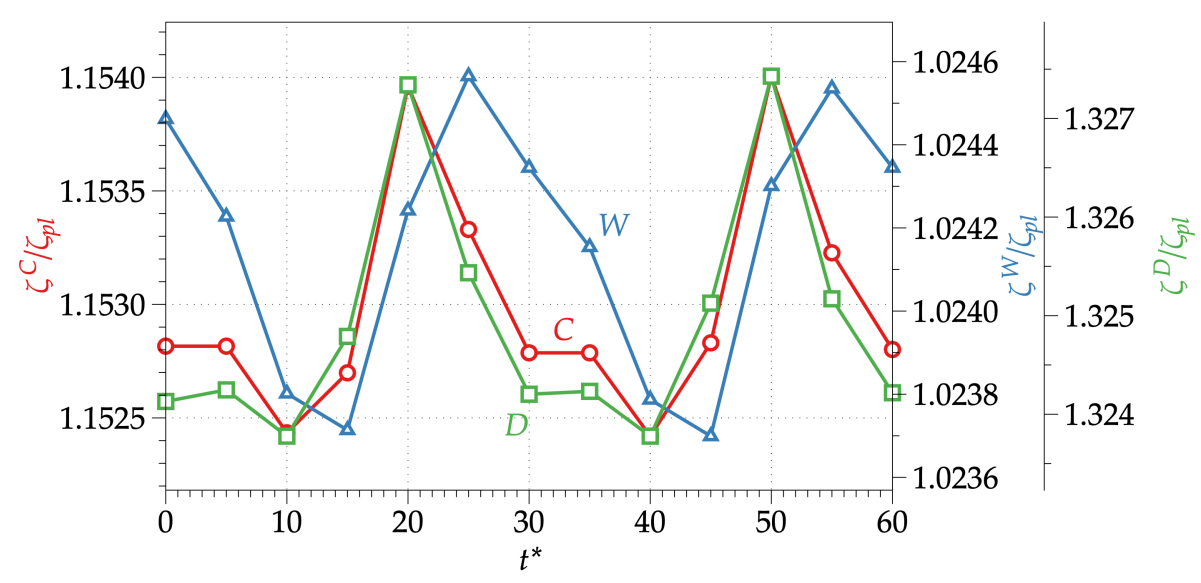

(b)

Figure 7. Dependences on $t^{*}$ of the thermal efficiency $N u_{m m} / N u_{m m_{p l}}(\mathbf{a})$ for the control section with the dimple (index C), the section in the wake behind the dimple (index W) and the section bounded by the dimple contour (index D), as well as (b) for the relative hydraulic losses in the channel in the control section (index $\mathrm{C}$ ), the area in the near wake behind the dimple (index $\mathrm{W}$ ) and the area bounded by the contour of the dimple (index D). 
The time dependences of the maximum increments of the dependent variables in the considered computational domain in the self-oscillating mode, as well as the integral force and thermal characteristics on the control section of the channel wall with a $2.5 \times 1.5$ dimple with a downstream center displacement of 0.5, shown in Figure 2 show the yield of aeromechanics processes and heat transfer in the dimpled channel for a periodic mode of change.

The periodicity of the change in the increments of the dependent variables in Figure $2 \mathrm{a}$ is reflected in the cyclicality of geometric figures shaded with different colors in the time interval from 300 to 400 . This interval corresponds to the self-oscillatory nature of the change in the transverse and frontal forces acting on the control section with a dimple, as well as the total heat transfer $N u_{m m}$. It is interesting to note that the release of the self-oscillating mode takes a rather long period of time-about 200-250 times from the state of rest in the conical dimple.

The period of oscillations that the periodic process of changing the vortex pattern and heat transfer in a channel with a deep conical dimple reaches is about 60 , which corresponds to a Strouhal number of about 0.016. It is interesting to note that the extreme values of the shear force $R_{z}$ acting on the control section in the self-oscillating mode turn out to be of the order of $5 \times 10^{-5}$, and the longitudinal force $R_{x}$ and integral heat transfer $N u_{m m}$, referred to the average values over the period of $R_{z}$ change, perform periodic oscillations in the range 0.005 .

Figure 3 compares the patterns of air spreading over the surface of the heated wall of the channel with a conical dimple, the static pressure field, dimensioned by double velocity head $\rho V^{2}$ and the jet-vortex structures in the dimple corresponding to two times at which $R_{z}$ takes extreme values.

The pictures reflect the extreme phases of the self-oscillating hydrodynamic process with the left- and right-sided flow structures in the dimple. The patterns of air spreading in Figure $3 \mathrm{a}, \mathrm{b}$ are displayed during computer visualization of streamlines in the near-wall layer of computational cells with a thickness of $10^{-4}$, indicating the direction of movement of liquid particles in the same way as it is done in [1-6]. As is known, such computer patterns correspond to the patterns of physical visualization of a near-wall vortex and separated flows obtained using soot-oil coatings, such as, for example, in [12,13].

The pictures presented in Figure 3 demonstrate two-cell vortex structures. On the side slopes of the dimple and on the bottom in the spreading patterns, special points such as foci are formed, in which tornado-like swirling jet streams are generated. The outer channel flow enters the dimple on the windward slope and spreads along the side slopes. In the case of $R_{z}=R z_{\text {min }}$, the zone of increased static pressure with a maximum equal to 0.12 is located to the left of the median plane of symmetry of the dimple, and when $R_{z}=R z_{\text {max }}$, it is to the right (Figure 3c,d). In the first case, when the air spreads, its transfer from the left half of the dimple to the right is noted. In the second case, on the contrary, when the air spreads, it moves from the right half of the dimple to the left. It should be emphasized that the zone of negative pressure with a minimum of -0.22 in both cases is located behind the edge of the dimple in the vicinity of the median longitudinal plane of symmetry.

Visualization of a jet tornado-like swirling flow in the dimple is performed by introducing a tape formed by labeled liquid particles in the vicinity of special points, such as foci on the left (red) and right (blue) slopes of the conical dimple (Figure 3e,f). In the case of $R_{z_{\text {min }}}$, a jet is generated in the dimple, spreading at an angle of $45^{\circ}$ from left to right, with the flow out of the dimple on the windward right slope. For $R_{z_{\text {max }}}$, the generated jet is inclined at an angle of $45^{\circ}$ from right to left, and the air flow swirling in it comes out on the left windward slope of the dimple. Thus, the two-cell vortex structure in the dimple is periodically rearranged with a change in the inclination of the transported swirling flow from left-to-right to right-to-left and back during the half-period of the self-oscillating flow regime.

It is interesting to note that the pattern of air spreading over the bottom of the dimple is consistent with the structure of swirling jet streams in the dimple. 
The distributions friction $f$ (made non-dimensional by twice the velocity head, $r h o V^{2}$ ), Nusselt number and temperature $T_{w}$ (non-dimensional with respect to $T_{r e f}$ ) in the longitudinal and transverse median sections of the conical dimple are compared at an oscillation period $R_{z}$ equal to 60 (Figure 4).

The curves are plotted in 10 steps in time. The self-oscillating nature of the flow around the conical dimple is illustrated, first of all, by the cyclic changes of the considered characteristics in the middle cross-section. Extreme values of dimensioned friction, Nusselt numbers and dimensioned temperatures periodically shift from right to left and back. It is interesting to note that the oscillating zone of intense reverse flow with minimal friction is rather narrow and is characterized by absolute values 2.5 times greater than the friction in the adjacent areas of the dimple bottom. It should also be emphasized that the maximum values of friction and Nusselt numbers are achieved in the near-edge windward zone of the dimple and in the area of conjugation of the bottom and the windward slope of the dimple at $t^{*}=10$ and 50 .

At the same time, the temperature decreases in the region of the windward edge and slightly increases at the point where the bottom meets the leeward slope.

The oscillation of the local extreme values of the velocity components of the return and secondary flow in the conical dimple at the oscillation period $R_{z}$ is shown in Figure 5. It is interesting to note that $-U_{\min }$ changes insignificantly (in the range of $0.369-0.373$ ), while $W_{\max }$ and $-W_{\min }$ are inferior in magnitude by $1.5^{-2}$ (the range of fluctuations is from 0.205 to 0.25$)$.

Comparison of the temperature distribution on the smooth wall of the plane-parallel channel and the temperature averaged over the oscillation period $R_{z}$ on the dimpled wall in Figure 6 shows the formation of temperature fields symmetric with respect to the median longitudinal section of the channel. The maximum temperature values on the surface of the dimple are significantly higher than the maximum temperature in the plane-parallel channel (about $2 \%$ ). The zones of maximum temperature are located on the side slopes of the dimple in the vicinity of the centers of generation of tornado-like jet-vortex structures.

Figure 7 compares the results on thermal efficiency for a rectangular control section with a conical dimple, a square section in the wake behind the dimple with a size of $1.5 \times 1.5$ and a section bounded by the contour of the dimple, as well as the results for relative hydraulic losses in the channel in the control section, a section in the near wake behind the dimple and the area bounded by the contour of the dimple at the oscillation period $R_{z}$. The hydraulic losses in a narrow channel with a conical dimple are calculated according to the method presented in [34].

The evaluation of the thermal efficiency of the dimpled channel in relation to the heated wall of a smooth plane-parallel channel demonstrates a periodic change in $N u_{m m} / N u_{m m_{p l}}$ in time over the period of oscillations $R_{z}$. The thermal efficiency values are fundamentally dependent on the heated wall section. On the control plot with a dimple, the range of variation of the relative Nusselt numbers is rather narrow and amounts to 1.035-1.041 (about 4\% increase). It is interesting to note that in the near wake behind a conical dimple in a square area, the thermal efficiency significantly (about 19\%) increases and changes in the range 1.189-1.1935. At the same time, in the area inside the conical dimple, the thermal efficiency is much less than unity and varies within a small range of 0.5955-0.604.

The relative hydraulic losses in the control section in the channel with the dimple are quite significant in comparison with the thermal efficiency and vary in the range 1.1525-1.154. In the area of the flat wall in the near wake behind the dimple, the relative hydraulic losses are small and vary in the range 1.0237-1.0246. It is interesting to emphasize that the thermal-hydraulic efficiency of the area in the near wake behind the dimple significantly exceeds 1 and averages about 1.16. The relative hydraulic losses of the channel section surrounding the conical dimple are large and vary in the range 1.324-1.3275. 


\section{Conclusions}

Numerical modeling of the periodic quasi-switching regime of vortex generation and heat transfer in a deep conical dimple with a slope angle of $10^{\circ}$ on the heated wall of a narrow channel was carried out on the basis of solving the unsteady Reynolds-averaged Navier-Stokes equations (URANS). When calculating the turbulent regime of the periodic one, the Menter 2003 model of the transfer of shear stresses, modified by taking into account the influence of the curvature of streamlines within the framework of the RodiLeshtsiner-Isaev approach, was used. Setting the fully developed air flow in the channel and the absence of air movement in the dimple as an initial approximation, the unsteady problem of reaching the self-oscillating flow regime in the dimpled channel with a periodic change in the local and integral characteristics of the flow and heat transfer, in particular, with a cyclic change in the transverse load $R_{z}$, was solved. Computer visualization of patterns of air spreading over the dimple surface did not demonstrate the formation of foci-type sources on the side faces of the dimple and the switching nature of the flow around the bottom. The identification of swirling jet-vortex flows inside the dimple by the method of tagged particles shows that a two-cell vortex system with different intensities of inclined swirling jet flows at the oscillation period $R_{z}$ is formed in the self-oscillatory mode. In this case, the angle of inclination of the jets changes in the range of $+/-45^{\circ}$. At $R e=10^{4}$, the oscillation period of the transverse $R_{z}$ and longitudinal forces $R_{x}$, as well as the total heat transfer $N u_{m m}$ to the control section of the heated channel wall with a dimple, was set equal to 60, which corresponds to the Strouhal number $S t=0.0167$. Periodic changes in friction, Nusselt numbers and temperature were recorded in the longitudinal and transverse median sections of the dimple and reflect the oscillations of the vortex structure from left to right and from right to left. The formation of a fan jet is shown, which oscillates relative to the plane of longitudinal symmetry, causing a redistribution of power and thermal loads. In general, fluctuations of local extreme and integral characteristics during the period of self-oscillations were small. There was also a zone of high thermal efficiency behind the conical dimple, the growth of the total averaged heat transfer in which it reaches $20 \%$, while hydraulic losses increase extremely insignificantly and do not exceed $2 \%$.

Author Contributions: Conceptualization, S.I. and A.S.; methodology, S.I.; software, A.S.; validation, N.T.; investigation, L.I.; resources, V.K.; data curation, A.U.; writing—original draft preparation, S.I.; writing-review and editing, N.T.; visualization, A.E. and N.T.; project administration, D.N. All authors have read and agreed to the published version of the manuscript.

Funding: The research was funded by the Ministry of Education and Science of the Russian Federation within the framework of the Program of the World-Class Science Center "Advanced Digital Technologies" (agreement dated 16 November 2020, No. 075-15-2020-903).

Conflicts of Interest: The authors declare no conflict of interest.

\section{References}

1. Iaccarino, G.; Durbin, P. Unsteady 3D RANS Simulations Using the v2-f Model; Technical Report; Center for Turbulence Research, Stanford University: Stanford, CA, USA, 2000.

2. Iaccarino, G.; Ooi, A.; Durbin, P.; Behnia, M. Reynolds averaged simulation of unsteady separated flow. Int. J. Heat Fluid Flow 2003, 24, 147-156. [CrossRef]

3. Isaev, S.; Lysenko, D. Calculation of unsteady flow past a cube on the wall of a narrow channel using URANS and the Spalart-Allmaras turbulence model. J. Eng. Phys. Thermophys. 2009, 82, 488-495. [CrossRef]

4. Isaev, S.; Baranov, P.; Usachov, A. Multiblock Computational Technologies in the VP2/3 Package on Aerothermo-Dynamics; LAP LAMBERT Academic Publishing: Saarbrucken, Germany, 2013; 316p.

5. Guvernyuk, S.; Egorichev, O.; Isaev, S.; Kornev, N.; Poddaeva, O. Numerical and physical simulation of wind influence on group of high-altitude buildings. Proc. Mosc. State Univ. Civ. Eng. 2011, 3, 185-191.

6. Isaev, S.A.; Baranov, P.A.; Zhukova, Y.V.; Tereshkin, A.A.; Usachov, A.E. Simulation of the Wind Effect on an Ensemble of High-Rise Buildings by means of Multiblock Computational Technologies. J. Eng. Phys. Thermophys. 2014, 87, 112-123. [CrossRef]

7. Rodi, W.; Ferziger, J.H.; Breuer, M.; Pourquié, M. Status of Large Eddy Simulation: Results of a Workshop. J. Fluids Eng. 1997, 119, 248-262. [CrossRef] 
8. Shah, K.B.; Ferziger, J.H. A fluid mechanicians view of wind engineering: Large eddy simulation of flow past a cubic obstacle. Comput. Wind. Eng. 1997, 67-68, 211-224. [CrossRef]

9. Shah, K.B. Large Eddy Simulations of Flow Past a Cubic Obstacle. Ph.D. Thesis, Stanford University, Stanford, CA, USA, 1998.

10. Krajnovic, S.; Davidson, L. Large eddy simulation of the flow around a surface mounted cube using a dynamic one-equation subgrid model. In First Symposium on Turbulence and Shear Flow Phenomena; TSFP Digital Library Online: Santa Barbara, CA, USA, 1999; pp. 741-746.

11. Yakhot, A.; Liu, H.; Nikitin, N. Turbulent flow around a wall-mounted cube: A direct numerical simulation. Int. J. Heat Fluid Flow 2006, 27, 994-1009. [CrossRef]

12. Hussein, H.J.; Martinuzzi, R.J. Energy balance for turbulent flow around a surface mounted cube placed in a channel. Phys. Fluids 1996, 8, 764-780 . [CrossRef]

13. Martinuzzi, R.; Tropea, C. The Flow Around Surface-Mounted, Prismatic Obstacles Placed in a Fully Developed Channel Flow (Data Bank Contribution). J. Fluids Eng. 1993, 115, 85-92. [CrossRef]

14. Isaev, S.; Baranov, P.; Kudryavtsev, N.; Lysenko, D.; Usachev, A. Comparative analysis of the calculation data on an unsteady flow around a circular cylinder obtained using the VP2/3 and fluent packages and the Spalart-Allmaras and Menter turbulence models. J. Eng. Phys. Thermophys. 2005, 78, 1199-1213. [CrossRef]

15. Snedeker, R.S.; Donaldson, C.D. Observation of a bistable flow in a hemispherical cavity. AIAA J. 1966, 4, 735-736. [CrossRef]

16. Terekhov, V.I.; Kalinina, S.V.; Mshvidobadze, Y.M. Heat Transfer Coefficient and Aerodynamic Resistance on a Surface with a Single Dimple. J. Enhanc. Heat Transf. 1997, 4, 131-145. [CrossRef]

17. Turnow, J.; Kornev, N.; Zhdanov, V.; Hassel, E. Flow structures and heat transfer on dimples in a staggered arrangement. In Proceedings of the 7th Symposium on Turbulence \& Shear Flow Phenomena (TSFP7), Ottawa, ON, Canada, 28-31 July 2011; Volume 35, pp. 168-175 [CrossRef]

18. Isaev, S.; Nikushchenko, D.; Sudakov, A.; Tryaskin, N.; Iunakov, L. Transformation of a separated turbulent flow in a conical dimple at the wall of a narrow channel and reduction in hydraulic losses as conicity increases. Tech. Phys. Lett. 2021, 47, 21-25. [CrossRef]

19. Ferziger, J.H.; Perić, M. Computational Methods for Fluid Dynamics, 2nd ed.; Springer: Berlin/Heidelberg, Germany, 1999.

20. Isaev, S.; Nikushchenko, D.; Sudakov, A.; Tryaskin, N.; Egorova, A.; Iunakov, L.; Usachov, A.; Kharchenko, V. Standard and Modified SST Models with the Consideration of the Streamline Curvature for Separated Flow Calculation in a Narrow Channel with a Conical Dimple on the Heated Wall. Energies 2021, 14, 5038. [CrossRef]

21. Menter, F.; Kuntz, M.; Langtry, R. Ten Years of Industrial Experience with the SST Turbulence Model. Heat Mass Transf. 2003, 4, 625-632.

22. Menter, F.; Ferreira, J.C.; Esch, T.; Konno, B. The SST Turbulence Model with Improved Wall Treatment for Heat Transfer Predictions in Gas Turbines. In Proceedings of the International Gas Turbine Congress, Tokyo, Japan, 2-7 November 2003.

23. Isaev, S.A. Experience of application of SST-model-2003 with correction on streamline curvature according to Rodi-LeshzinerIsaev approach for (U)RANS calculations of separated and vortex sub- and supersonic flows. AIP Conf. Proc. 2018, $2027,020015$. [CrossRef]

24. Launder, B.E.; Spalding, D.B. The numerical computation of turbulent flows. Comput. Methods Appl. Mech. Eng. 1974, 3, 269-289. [CrossRef]

25. Leschziner, M.; Rodi, W. Calculation of Annular and Twin Parallel Jets Using Various Discretization Schemes and TurbulenceModel Variations. J. Fluids Eng. 1981, 103, 352-360. [CrossRef]

26. Isaev, S.; Leontiev, A.; Chudnovsky, Y.; Nikushchenko, D.; Popov, I.; Sudakov, A. Simulation of Vortex Heat Transfer Enhancement in the Turbulent Water Flow in the Narrow Plane-Parallel Channel with an Inclined Oval-trench Dimple of Fixed Depth and Spot Area. Energies 2019, 12, 1296. [CrossRef]

27. Isaev, S.; Schelchkov, A.; Leontiev, A.; Gortyshov, Y.F.; Baranov, P.; Popov, I. Vortex heat transfer enhancement in the narrow plane-parallel channel with the oval-trench dimple of fixed depth and spot area. Int. J. Heat Mass Transf. 2017, 109, 40-62. [CrossRef]

28. Leonard, B. A stable and accurate convective modelling procedure based on quadratic upstream interpolation. Comput. Methods Appl. Mech. Eng. 1979, 19, 59-98. [CrossRef]

29. van Leer, B. Towards the ultimate conservative difference scheme. V. A second-order sequel to Godunov's method. J. Comput. Phys. 1979, 32, 101-136. [CrossRef]

30. Rhie, C.M.; Chow, W.L. Numerical study of the turbulent flow past an airfoil with trailing edge separation. AIAA J. 1983, 21, 1525-1532. [CrossRef]

31. Pascau, A.; Garcia-Polanco, N. Consistency of simplec scheme in collocated grids. In Proceedings of the Fifth European Conference on Computational Fluid Dynamics, Lisbon, Portugal, 14-17 June 2010.

32. Saad, Y. Iterative Methods for Sparse Linear Systems, 2nd ed.; Society for Industrial and Applied Mathematics: Philadelphia, PA, USA, 2003.

33. Demidov, D. AMGCL-A C++ library for efficient solution of large sparse linear systems. Softw. Impacts 2020, 6, 100037. [CrossRef]

34. Isaev, S.; Kornev, N.; Leontiev, A.; Hassel, E. Influence of the Reynolds number and the spherical dimple depth on turbulent heat transfer and hydraulic loss in a narrow channel. Int. J. Heat Mass Transf. 2010, 53, 178-197. [CrossRef] 